\title{
Emergency Tracking of the Heart Patients
}

\author{
Pooja P.Nemade ${ }^{1}$, Dr. G.A.Kulkarni ${ }^{2}$ \\ Student of M. Tech, Dept. of E\&TC, SSGB College of Engineering \& Technology, Bhusawal, Maharashtra, India ${ }^{1}$ \\ HOD of Dept. of E\&TC, SSGB College of Engineering \& Technology, Bhusawal, Maharashtra, India ${ }^{2}$
}

\begin{abstract}
The author is developing an ECG monitoring device that can be used by non-technical users in need of health monitoring and is integrated with a broader internet-of-things is being taken to a greater extent. Along with ECG monitoring it also sends the GPS location to the medical experts so that in case of urgent medical assistance the same can be reached out as soon as possible. The techniques used here are using discrete electronic components and a custom PCB. The live data can be received from the patient's body and will be transmitted at the health care where the GPS location of the patient can be traced by the doctor. We gave a thought to build this device so that the data collection can be stored at the doctor's end and it can also provide urgent medical attention without which patients can have fatal incidence. There are many similar devices in the market but ours is the cheapest ensuring even the common people can afford it. It aims to be used by the people in areas where medical assistance may not be readily available.
\end{abstract}

Keywords: Wireless ECG monitoring system, Wearable monitoring system, Bluetooth modem, USB, GPS tracker, android phone

\section{INTRODUCTION}

Recently, health monitoring systems are achieving higher significance as the rapidly growing universal elderly population increases the demand of caretakers. Almost in every single house we will find a person suffering from heart disease, diabetes, blood pressure and many more. The rate of heart patients is increasing day by day. So to make the check-up routines more easier for the patients and to reduce the death caused by heart attacks we can are implementing a technology where the patient can be monitored sitting at home and the doctors can track the location of the patient.

In India, the age-standardized annual stroke incidence rate is 154 per 100000 per year. In general, the stroke incidence and stroke-related case mortality rates in India are higher relative to Western industrialized nations, abnormally higher among women. Available neuroimaging data suggest that haemorrhagic strokes are more common in India than in the Western population. [3] The ratio of ischemic stroke to hemorrhagic stroke mortality in India is below the global average of close to 1 . In comparison with the western populations in India this imitates the proportionately less advanced stage of the epidemiological transition.

In the past 25 years, India has endorsed an alarming rise in the occurrence of heart disease, stroke, diabetes and cancers, a series of new studies published on Wednesday in The Lancetand its associated journals have revealed.[4]

From 1990 to 2016, in India, the widespread presence of heart disease and stroke has increased by over 50\%, with an increase observed in every state. [4] The noteworthy inclusion of these diseases to total disease and death are burden in the country has almost multiplied by two as of now, in the past 25 years. Now, heart disease is the major discrete cause of disease burden in India, and stroke is the fifth leading cause. [4] In India 2016, heart disease and stroke contributed to $28.1 \%$ of total deaths compared to $15.2 \%$ in 1990 . Where $17.8 \%$ of deaths were of heart disease and $7.1 \%$ deaths were due to stroke. In men's the deaths proportion for heart disease was higher than in women's but the death proportion for stroke were common in both men and women. [4] In USA the average stroke rate per second is $40 \mathrm{sec}$ [5]. In 2016, people died of stroke every 3 minutes 42 seconds. Stroke ranks on no.5 in US when considered separately from other cardiovascular diseases and kills approximately 142000 people per year. The age-adjusted stroke death rate was 37.3 per 100,000 in 2016 , a decrease of $16.7 \%$ from 2006 , although the actual number of stroke deaths increased $3.7 \%$ during the same time period[5].

In US, the data from 2005 displays that stroke was a leading cause of significant lasting disability. Roughly around 3\% of males and $2 \%$ of females reported that they were disabled due to stroke. As claimed by 2016 data, Eastern Europe, East Asia, and parts of Southeast Asia, Central Asia, and sub-Saharan Africa had the highest rates of stroke mortality. Age-reversal widely spread presence rates of stroke were higher in East Asia and Eastern Europe. In recent researches, communication and microelectronics protocols these factors enables performance upgradation and cost reduction at individual sensors level as well as at infrastructure level. Health monitoring is more a choice than a necessity for today's youth. So, to overcome and make this issue user friendly here we are trying to build a system where the patients of heart disease can purchase it at cheaper rate and can monitor their ECG whenever they want at any place. This data is directly transferred at the doctor's end through IOT. The main and the efficient factor is that if the patient is suffering a major stroke the doctor can easily trace the location of the patient. 
Vol. 8, Issue 5, May 2019

This paper is organised in the following sections:

Section II describes the overall system design and description of the project

Section III describes the discussion

Section IV describes the results and implementation part

Section V describes the conclusion, future scope.

\section{SYSTEM DESCRIPTION}

The designed block schematic of the ECG monitoring system is shown below:

The system is composed of 3 parts:

(i) Sensors : collects the data and analyse it from the human body

(ii) Controller : processes the collected data and stores into the memory

(iii) Android Phone : receives the controllers stored data using Bluetooth module at a time uploads the receiving data on the web server for remote access for the purpose of medical support.

(iv) GPS Location: the doctor can trace the location of the patient along with the ECG.

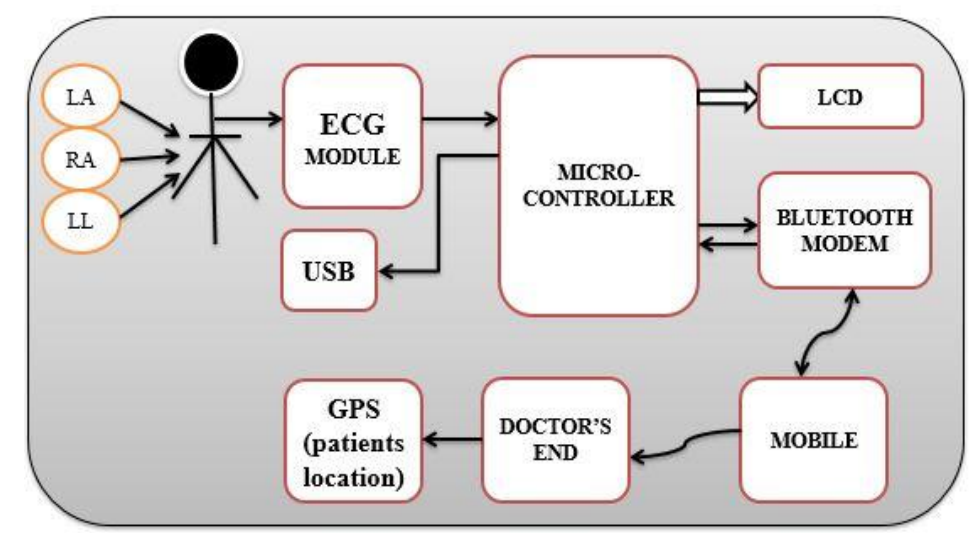

Figure 1: Block Schematic

\section{ECG module.}

The main function of the ECG module is to sense the signals from the human body and acts as a sensor. An external power supply is being added to the ECG and needs separate batteries for each sensor detection. 2 separate batteries of $9 \mathrm{~V}$ are being used here for high gain output. AD624 IC is being used here for detecting the signals. This high gain AD624 converts the microvolts signals into higher ranges and sends it to the controller. AD624 has very high gain, high input impedance, very high common mode rejection ratio. It is also used in industrial application and in test and measurement equipment's. As seen in the diagram below, we can see a small plunge with a large barb and another plunge. Both the dips and the spike together makes the QRS wave. The QRS waves are occasionally called as QRS complex and this represents the depolarization of the ventricles.

Now the measurement of the QRS intervals are being shown:

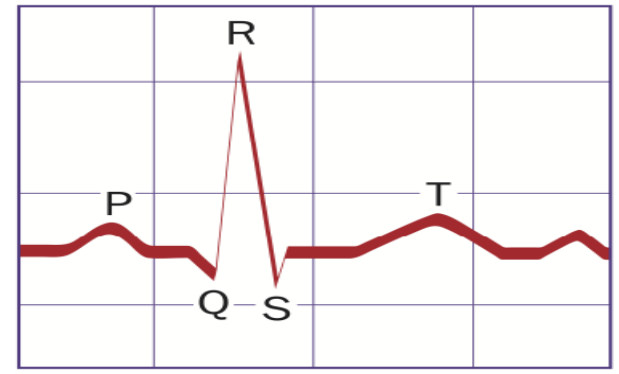

Figure No. : 2

The normal range of the person at rest is between $60-100$ beats per minute(bpm).

The calculation of QRS complex is shown below:

- $\quad \mathrm{Q}$ wave is the first downstroke of the QRS complex which is very small or absent. 
Vol. 8, Issue 5, May 2019

- $\quad \mathrm{R}$ wave is the first upward deflection of the QRS complex and upward deflections occurring after an $\mathrm{S}$ wave are noted by a "prime mark" such as R'

- $\quad \mathrm{S}$ wave is the first downward deflection occurring after the $\mathrm{R}$ wave.

ECG is carried out by placing the electrodes on the skin of the patient's heart. The voltage measurement between the electrode varies as the electrical impulse moves from the atria which are the topmost two chambers, and this produces the graph of your heart is performing. This provides us with the valuable information based on the time. Here we have used the instrumentation amplifier instead of differential amplifier but many times in industrial applications the location where the measurements are carried out and the location where the sensors are deployed are quite away from each other. So, in this case it is possible that the noise or the interference signal will get superimposed over the measured signal and to avoid the problem many times the signal is measured using the differential measurement technique. By using buffer circuits we can add along the IC's we can solve the problem if input impedance and mismatch between the resistor pairs. The problem with the monolithic IC are the gain and this is provided by the IC which is fixed because the gain cannot be changed. To avoid these non-inverting op-amps instead of buffer circuit. The initial gain can be provided by the non-inverting buffers. Problems along with the circuit is along with differential input signal and the common mode input signal will also be amplified so we will not get any improvement in the signal to noise ratio. To avoid these parameters we have used the Instrumentation amplifier. Only the differential input signal will get amplified with the opamps and the common mode signal will pass as it is and for this reason the instrumentation amplifier is available in IC format. As they are fabricated internally they are highly matched. Using the common gain Rg we can set the gain of the instrumentation amplifier.

The output is calculated using the formula:

$$
V 0=\frac{R 2}{R 1}\left(1+\frac{2 R 5}{R 4}\right)(V A-V B)
$$

The CMRR value can be further calculated using this equation. It is useful in environment where the differential input signal is very small and the common mode input signal which is present at the 2 terminal is larger than the differential input signal that is why the instrumentation amplifier is preferred over the differential amplifier.

\section{DISCUSSION}

\begin{tabular}{|l|l|l|}
\hline Author & Authors method & Drawback \\
\hline $\begin{array}{l}\text { Emil } \\
\text { Valchinov }\end{array}$ & $\begin{array}{l}\text { Measurements were performed with the ECG } \\
\text { system using compression vest providing firm } \\
\text { thoracic elastic enclosure }\end{array}$ & $\begin{array}{l}\text { Needs to improve the signal quality over the } \\
\text { skin perspiration, fabric and electrode materials. }\end{array}$ \\
\hline $\begin{array}{l}\text { Takuji } \\
\text { Suzuki }\end{array}$ & $\begin{array}{l}\text { Introduced Bluetooth transrecievers are } \\
\text { integrated into a } 14.5^{*} 14.5 \mathrm{~mm} \text { module }\end{array}$ & $\begin{array}{l}\text { Using this Bluetooth chips the noise factor is } \\
\text { present in the system so to reduce this factor the } \\
\text { system can be introduced in a advanced form }\end{array}$ \\
\hline Samuel & $\begin{array}{l}\text { Enables the android phone with the Bluetooth } \\
\text { to receive the incoming signal and send it to the } \\
\text { health care system using the circuitry of } \\
\text { transmission. }\end{array}$ & $\begin{array}{l}\text { The prototype could produce clear quality ECG } \\
\text { traces on the mobile screen when connected to } \\
\text { several individuals. }\end{array}$ \\
\hline $\begin{array}{l}\text { Eric } \\
\text { S.Winokur }\end{array}$ & $\begin{array}{l}\text { This system is developed from discrete } \\
\text { electronic components and a custom PCB }\end{array}$ & $\begin{array}{l}\text { Improvement in mechanical and electric } \\
\text { stability during high levels of activity and } \\
\text { increased memory size will allow the necessary } \\
\text { data for long term heath care analysis }\end{array}$ \\
\hline
\end{tabular}

\section{THE COMPARISON OF THE PREVIOUSLY DISCUSSED METHODS ARE CLASSIFIED BELOW}

Table no. 1: Comparison of the previous authors results.

\section{RESULTS AND IMPLEMENTATION}

Preliminary test results were performed using the ECG module. Using this module we have measured the ECG of a 7 years age group, 26 years age group, critical patient, normal patient, patient with heart disease. ECG have been monitored and sent to the doctor's end for the further monitoring of the patient. The GPS location of the patient is also being tracked at the same time in emergency case. The doctor's have recommended this module so that be used on the monthly basis by the patient. The table shown below mentions the drawbacks of the module earlier being developed. In comparison with these papers this circuit is user friendly to the patient, low cost and more efficient. The main factor is 
that the signal quality is being improved and the signal from the body is easily detected as compared to the previous modules. The main factor we have added is the GPS location.

The results in the form og GUI is shown below:

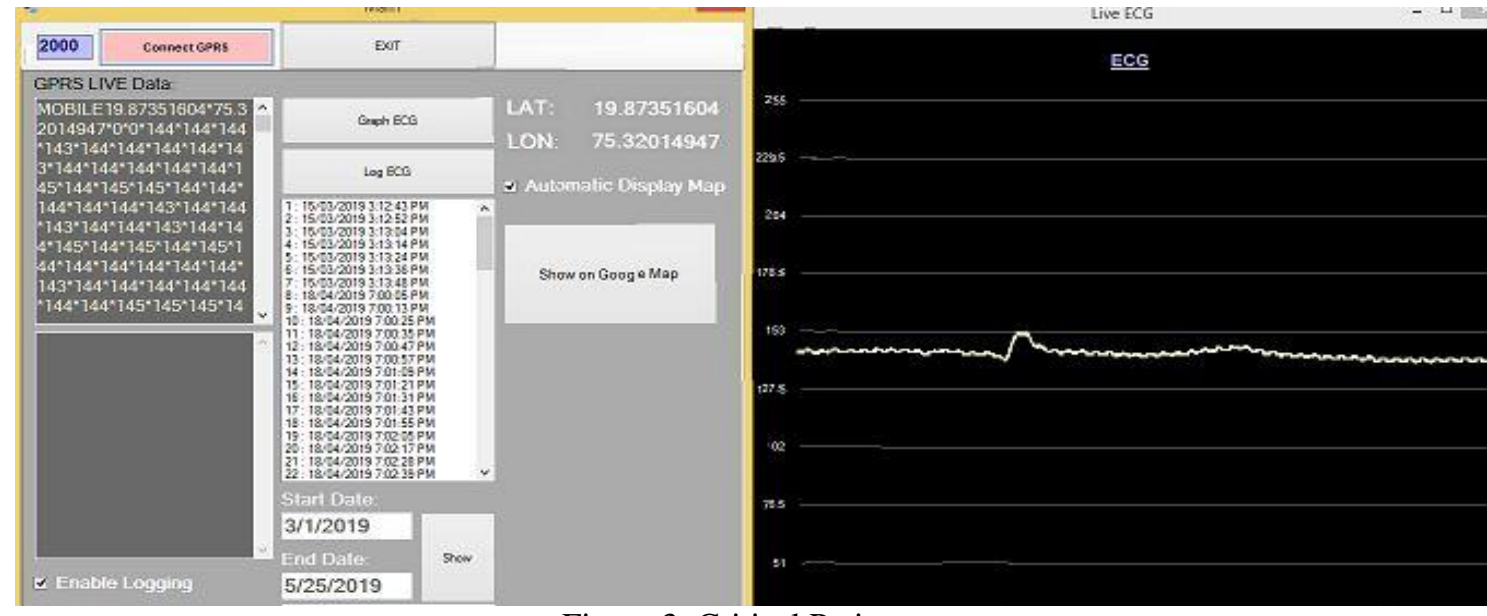

Figure 3: Critical Patient

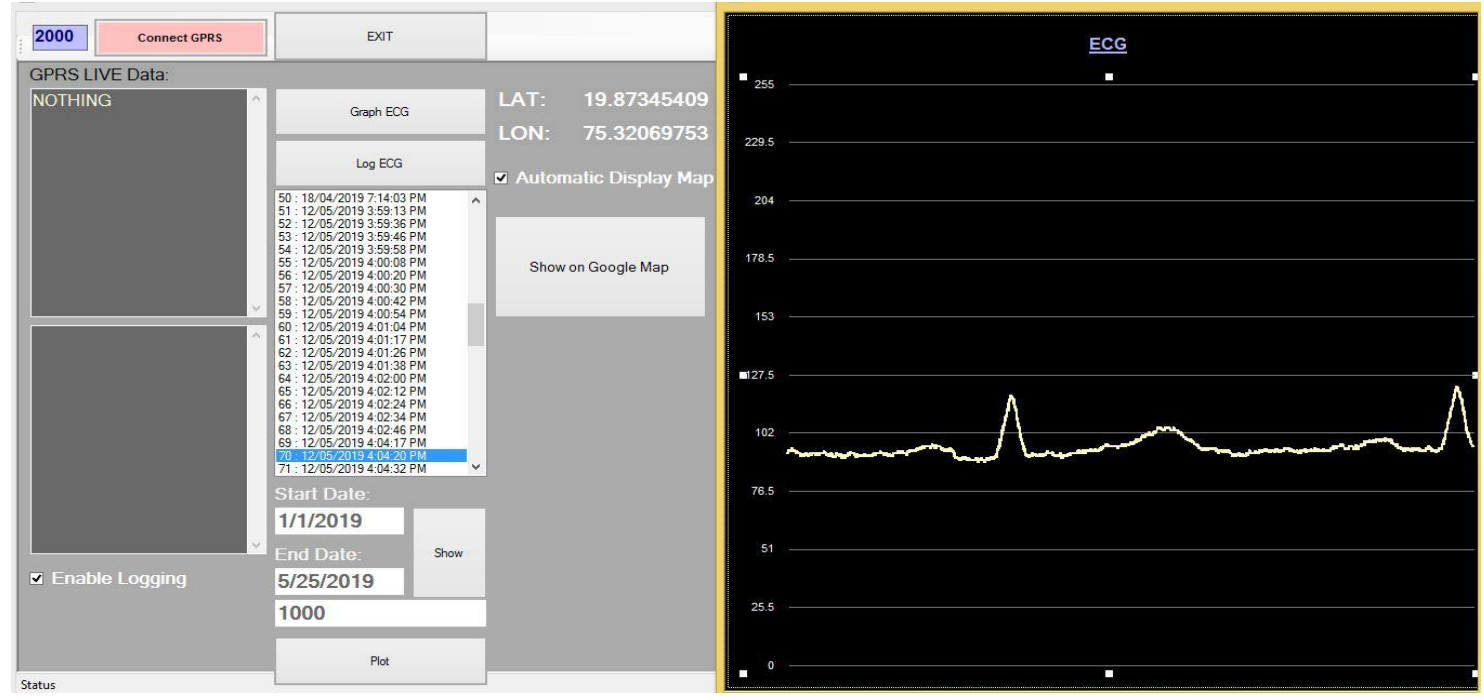

Figure 4: Normal patient

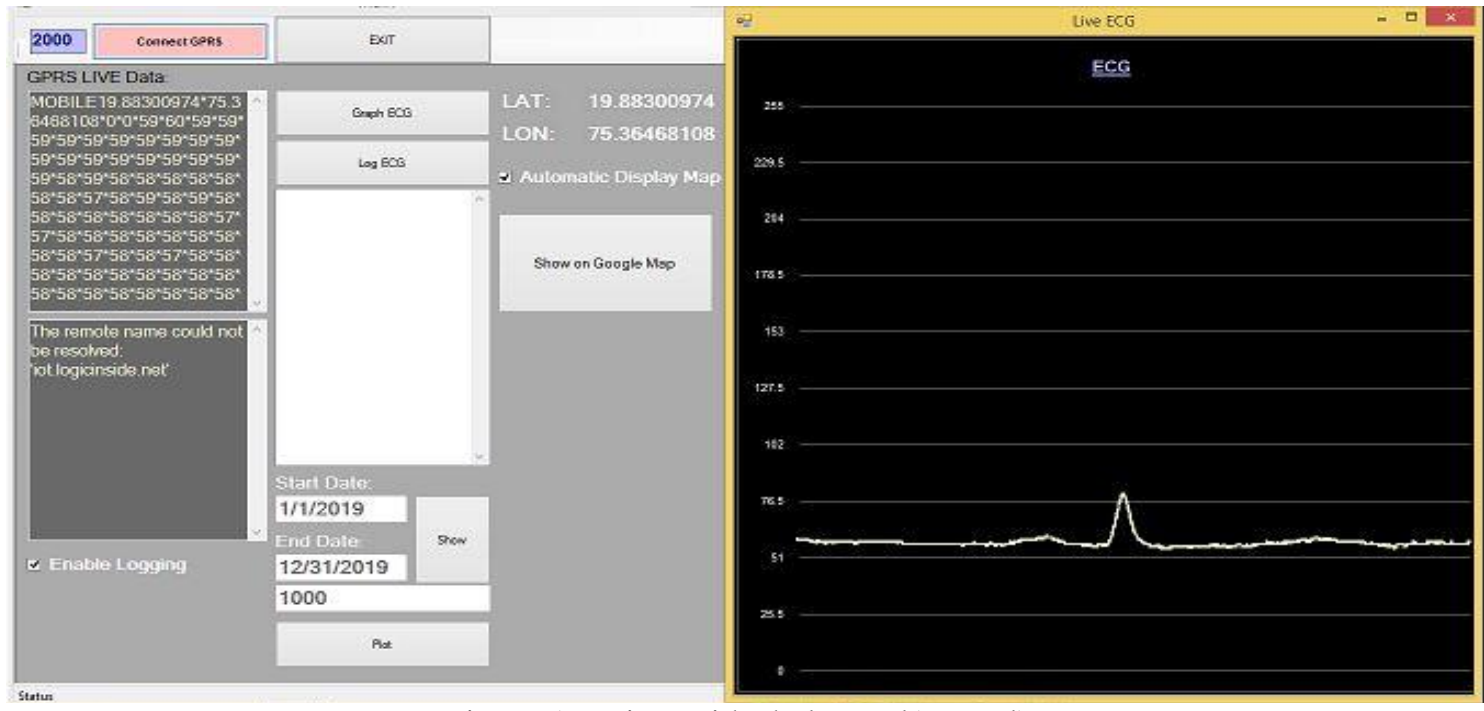

Figure 5: Patient with cholesterol(normal) 
Vol. 8, Issue 5, May 2019

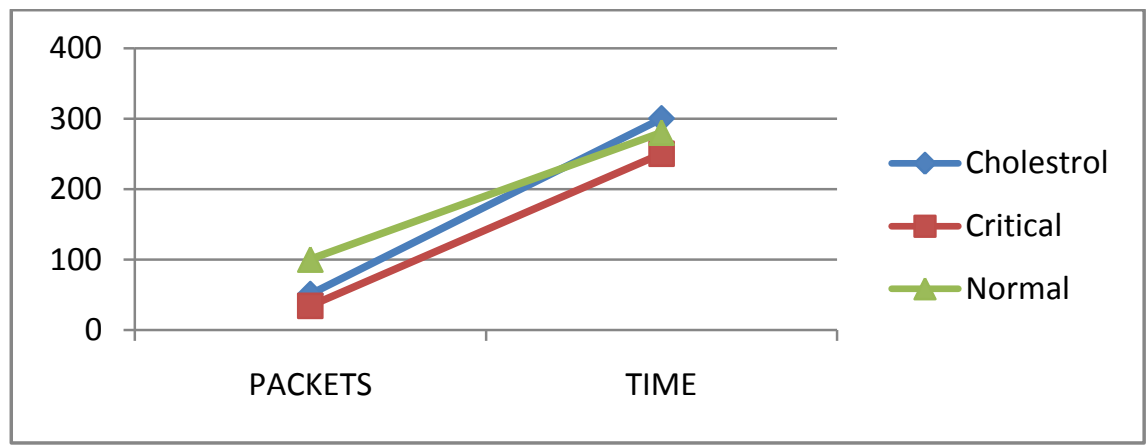

Figure 6: Linear graph of patients compared with their expected values.

\section{CONCLUSION}

We have developed this mechanism on an Internet-Of-Things platform to overcome the disadvantages of the current system. Our device can be handled by a layman with ease, it is cost-effective, accurate ECG graph, data is stored with time data n ECG graph, and accurate data collection shows the geographical location of the patient where medical help can be provided even when the patient is not in a condition to communicate his location. Our main aim is to make the lives of common man simpler and aid the medical profession in a minor but crucial way. Overall the good results achieved with the presented system provoked us to further bring up the project with the (i) implementation of the heart rate meter function (ii) implementation of 12-lead and display function to enable diagnostics on the heart muscle by a specialist. (iii) to enable automatic detection and warning of the ECG signal quality. (iv) deploying the artificial intelligence techniques to automatically warn and detect the heart threatening arrhythmias and cardiac conditions of the patient.

\section{REFERENCES}

[1]. https://www.livemint.com

[2]. https://en.wikipedia.org/wiki/Electrocardiography

[3]. https://www.thehindubusinessline.com/news/science/heart-disease-stroke-among-top-killers-in-india/article24935985.ece

[4]. https://www.ahajournals.org/doi/full/10.1161/CIRCULATIONAHA.114.008729

[5]. https://healthmetrics.heart.org/wp-content/uploads/2019/02/At-A-Glance-Heart-Disease-and-Stroke-Statistics-\%E2\%80\%93-2019.pdf

[6]. https://www.downtoearth.org.in/news/health/heart-disease-stroke-among-top-killers-in-india-61602

[7]. Karandeep Malhi; Subhas Chandra Mukhopadhyay; "Julia Schnepper et al.(2012) A Zigbee-Based Wearable Physiological Parameters Monitoring System", IEEE Sensors Journal( Volume: 12 , Issue: 3 , March 2012 )

[8]. Hyejung Kim; Sunyoung Kim ; Nick Van Helleputte; Antonio Artes et al.: "A Configurable and Low-Power Mixed Signal SoC for Portable ECG Monitoring Applications", IEEE Transactions on Biomedical Circuits and Systems ( Volume: 8 , Issue: 2 , April 2014 )

[9]. Reza S. Dilmaghani; Hossein Bobarshad; M. Ghavami; “Wireless Sensor Networks for Monitoring Physiological Signals of Multiple Patients", IEEE Transactions on Biomedical Circuits and Systems (Volume: 5 , Issue: 4 , Aug. 2011 )

[10]. Emil Valchinov; Athanasios Antoniou ; Konstantinos Rotas : et al. "Wearable ECG system for health and sports monitoring",2014 4th International Conference on Wireless Mobile Communication and Healthcare - Transforming Healthcare Through Innovations in Mobile and Wireless Technologies (MOBIHEALTH)

[11]. Juan Pablo Tello ; Oscar Manjarres ; Mauricio Quijano ; "Remote Monitoring System of ECG and Human Body Temperature Signals", IEEE Latin America Transactions (Volume: 11 , Issue: 1, Feb. 2013 )

[12]. Yuan Wang; Yiping Zheng; Ou Bai ; Qisong Wang; "A multifunctional wireless body area sensors network with real time embedded data analysis", 2016 IEEE Biomedical Circuits and Systems Conference (BioCAS)

[13]. Maradugu Anil Kumar; Y.Ravi Sekhar, "Android based health care monitoring system", 2015 International Conference on Innovations in Information, Embedded and Communication Systems (ICIIECS)

[14]. Elisa Spanò ; Stefano Di Pascoli; Giuseppe Iannaccone, “ Low-Power Wearable ECG Monitoring System for Multiple-Patient Remote Monitoring”, IEEE Sensors Journal ( Volume: 16, Issue: 13 , July1, 2016 )

[15]. Bayilmis C, Younis M (2012) Energy-aware gateway selection for increasing the lifetime of wireless body area sensor networks. J Med Syst 36(3):1593-1601.

[16]. Bodin O, Loginov D, Mitrokhina N (2008) Improvement of ECG analysis for monitoring of cardiac electrical activity. Biomed Eng 42(3):128131.

[17]. Chatterjee HK, Gupta R, Mitra M (2011) A statistical approach for determination of time plane features from digitized ECG. Comput Biol Med 41(5):278-284

[18]. Kaufmann T, Su“tterlin S, Schulz S, Vo“gele C (2011) ARTii-FACT: a tool for heart rate artifact processing and heart rate variability analysis. Behav Res Methods 43(4):1161-1170

[19]. Merzougui R, Feham M, Sedjelmaci H (2011) Design and implementation of an algorithm for cardiac pathologies detec-tion on mobile phone. Int J Wireless Inf Netw 18(1):11-23

[20]. Shin W, Cha Y, Yoon G (2010) ECG/PPG integer signal pro-cessing for a ubiquitous health monitoring system. J Med Syst 34(5):891-898.

[21]. Yan J, Lu Y, Liu J, Wu X, Xu Y (2010) Self-adaptive model-based ECG denoising using features extracted by mean shift algorithm. Biomed Signal Process Control 5(2):103-113

[22]. Wu Y, Rangayyan RM, Zhou Y, Ng S-C (2009) Filtering electrocardiographic signals using an unbiased and normal-ized adaptive noise reduction system. Med Eng Phys 31(1): 17-26

[23]. Puurtinen M, Viik J, Hyttinen J (2009) Best electrode locations for a small bipolar ECG device: signal strength analysis of clinical data. Ann Biomed Eng 37(2):331-336. 


\title{
International Journal of Advanced Research in Computer and Communication Engineering
}

\author{
Vol. 8, Issue 5, May 2019
}

[24]. Sankari Z, Adeli H (2011) HeartSaver: a mobile cardiac moni-toring system for auto-detection of atrial fibrillation, myocardial infarction, and atrio-ventricular block. Comput Biol Med 41(4):211-220

[25]. Scherr D, Dalal D, Henrikson C, Spragg D, Berger R, Calkins H, Cheng A (2008) Prospective comparison of the diagnostic utility of a standard event monitor versus a "leadless" portable ECG monitor in the evaluation of patients with palpitations. J Interv Cardiac Electrophysiol 22(1):39-44.

[26]. Winterhalter M, Schiller J, Münte S, Bund M, Hoy L, Weilbach C, Piepenbrock S, Rahe-Meyer N (2008) Prospective investi-gation into the influence of various stressors on skin impedance. J Clin Monit Comput 22(1):67-74

[27]. Mamaghanian H, Khaled N, Atienza D, Vandergheynst P (2011) Compressed sensing for real-time energy-efficient ECG com-pression on wireless body sensor nodes. IEEE Trans Biomed Eng 58(9):2456-2466

[28]. Oresko JJ, Zhanpeng J, Jun C, Shimeng H, Yuwen S, Duschl H, Cheng AC (2010) A Wearable smartphone-based platform for real-time cardiovascular disease detection via electrocardiogram processing. IEEE Trans Inf Technol Biomed 14(3):734-740

[29]. Chin-Teng L, Kuan-Cheng C, Chun-Ling L, Chia-Cheng C, Shao-Wei L, Shih-Sheng C, Bor-Shyh L, Hsin-Yueh L, Ray-Jade C, Yuan-Teh L, Li-Wei K (2010) An intelligent telecardiology system using a wearable and wireless ECG to detect atrial fibrillation. IEEE Trans Inf Technol Biomed 14(3):726-733

[30]. Chung-Chih L, Ping-Yeh L, Po-Kuan L, Guan-Yu H, Wei-Lun L, Ren-Guey L (2008) A healthcare integration system for dis-ease assessment and safety monitoring of dementia patients. IEEE Trans Inf Technol Biomed 12(5):579-586

[31]. Kim M, Cho Y, Seo S-T, Son C-S, Kim Y-N (2011) A new method of ECG feature detection based on combined wavelet transform for u-health service. Biomed Eng Lett 1(2):108-115.

[32]. Bsoul M, Minn H, Tamil L (2011) Apnea MedAssist: real-time sleep apnea monitor using single-lead ECG. IEEE Trans Inf Technol Biomed 15(3):416-427

[33]. Yoo J, Long Y, Seulki L, Hyejung K, Binhee K, Hoi-Jun Y (2009) An Attachable ECG sensor bandage with planar-fashionable circuit board. In: Ferscha A, Stocker G (eds) Inter-national Symposium on Wearable Computers, 2009. ISWC '09, Linz, Austria, 4-7 Sept 2009. IEEE Computer Society, Con-ference Publishing Services (CPS), pp 145-146

[34]. Yoon Y, Cho J, Yoon G (2009) Non-constrained blood pressure monitoring using ECG and PPG for personal healthcare. J Med Syst 33(4):261266.

[35]. Yoo J, Long Y, Seulki L, Hyejung K, Hoi-Jun Y (2009) A wearable ECG acquisition system with compact planar-fash-ionable circuit boardbased shirt. IEEE Trans Inf Technol Bio-med 13(6):897-902

[36]. U-j Yoon, Noh Y-S, H-r Yoon (2011) Optimization methods for improving the performance of heart rate detection by a wearable ECG system during high-intensity exercise. Biomed Eng Lett 1(2):143-150

[37]. Pollonini L, Rajan N, Xu S, Madala S, Dacso C (2012) A novel handheld device for use in remote patient monitoring of heart failure patientsdesign and preliminary validation on healthy subjects. J Med Syst 36(2):653-659.

[38]. Sardini E, Serpelloni M (2010) Instrumented wearable belt for wireless health monitoring. Procedia Eng 5:580-583

[39]. Jourand P, De Clercq H, Corthout R, Puers R (2009) Textile integrated breathing and ECG monitoring system. Procedia Chem 1(1):722-725

[40]. Lee Y-D, Chung W-Y (2009) Wireless sensor network based wearable smart shirt for ubiquitous health and activity moni-toring. Sensors Actuators B Chemical 140(2):390-395

[41]. Lo'pez G, Custodio V, Moreno JI (2010) LOBIN: E-textile and wireless-sensor-network-based platform for healthcare moni-toring in future hospital environments. IEEE Trans Inf Technol Biomed 14(6):1446-1458

[42]. Heilman K, Handelman M, Lewis G, Porges S (2008) Accuracy of the StressEraser in the detection of cardiac rhythms. Appl Psycho-physiol Biofeedback 33(2):83-89

[43]. Bianchi AM, Mendez MO, Cerutti S (2010) Processing of sig-nals recorded through smart devices: sleep-quality assessment. IEEE Trans Inf Technol Biomed 14(3):741-747

[44]. Buttussi F, Chittaro L (2008) MOPET: a context-aware and user-adaptive wearable system for fitness training. Artif Intell Med 42(2):153-163

[45]. Chang H-T, Chung C-G, Chen M-W (2011) An e-caring chair for physiological signal measurement and recording. Med Eng Phys 35(2):277282

[46]. Coyle S, King-Tong L, Moyna N, O’Gorman D, Diamond D, Di Francesco F, Costanzo D, Salvo P, Trivella MG, De Rossi DE, Taccini N, Paradiso R, Porchet JA, Ridolfi A, Luprano J, Chuzel C, Lanier T, Revol-Cavalier F, Schoumacker S, Mourier V, Chartier I, Convert R, DeMoncuit H, Bini C (2010) BIO-TEX-biosensing textiles for personalised healthcare manage-ment. IEEE Trans Inf Technol Biomed 14(2):364370

[47]. McFee R, Baule GM (1972) Research in electrocardiography and magnetocardiography. In: Rowe JE (ed) Proceedings of the IEEE, USA, 1972, vol 3. The Institute of Electrical and Electronics Engineers, Inc., pp 290-32

[48]. Yanowitz F, Kinias P, Rawling D, Fozzard HA (1974) Accuracy of a continuous real-time ECG dysrhythmia monitoring system. Circulation 50(1):65-72

[49]. Nyga ${ }^{\circ}$ ds M-E, Hulting J (1979) An automated system for ECG monitoring. Comput Biomed Res 12(2):181-202

[50]. Pahlm O, So“rnmo L (1984) Software QRS detection in ambu-latory monitoring-a review. Med Biol Eng Comput 22(4):289-297.

[51]. Kleinmuntz B (1984) Diagnostic problem solving by computer: a historical review and the current state of the science. Comput Biol Med 14(3):255-270 